\title{
Becoming a mother was a difficult, multifactorial process
}

Barclay L, Everitt L, Rogan F, et al. Becoming a mother-an analysis of women's experience of early motherhood.J Adv Nurs 1997 Apr;25:719-28.

Rogan F, Schmied V, Barclay L, et al. 'Becoming a mother'-developing a new theory of early motherhood.J Adv Nurs 1997 May;25:877-85.

\section{Objective}

To describe women's experiences of early motherhood.

\section{Design}

Grounded theory.

\section{Setting}

South Eastern Sydney Area Health Service, Australia.

\section{Participants}

55 first time mothers from 23-39 years old. 67\% were previously employed in clerical, secretarial, or manual jobs. Infants were of similar age (mean age 12 weeks, range 2-26). Fathers were mainly employed in manual trades $(62 \%)$.

\section{Methods}

9 focus groups were held. Open ended questions were used to trigger discussions which were tape recorded and transcribed. The constant comparative method was used to analyse 5 of the transcripts until theoretical saturation was reached. Emerging concepts were categorised according to their characteristics and the relation between them. The remaining 4 transcripts confirmed the concepts and resulting categories, which were verified by another group of new mothers and experienced health professionals.

\section{Main results}

Becoming a mother has 6 categories. The first is realising the overwhelming process of becoming a mother and the consequences this has on one's life. Second, comes the feeling of being unready for the reality of motherhood. Third is feeling drained from the physical, mental, and emotional demands of the new role, and the fourth is feeling alone, unsupported, and anxious. Fifth is a feeling of loss of time, freedom, independence, control, and self. Sixth, women feel that they have worked these issues out by developing skills and gaining confidence in being mothers.

3 factors influenced women's experience of new motherhood. Firstly, the mother's perception of the nature of her baby influenced her perception of herself as a mother; secondly, previous experience with babies helped the mothers feel more prepared for the process of becoming a mother; thirdly, the availability of social support was crucial.

\section{Conclusions}

Becoming a mother was a difficult, multifactorial process. The 6 categories involved in becoming a mother and the 3 factors that influence women's experience of new motherhood show the complexity of this experience.

Source of funding: Department of Employment, Education, and Training Research Infrastructure Grant, Australia.

For article reprint:Professor L Barclay, Family Health Unit, St. George Hospital, Gray Street, Kogarah, New South Wales 2217, Australia. Fax +61 293503976.

\section{Commentary}

This study by Barclay et al outlines a complex process that first time mothers experience in adjusting to their new role. Strengths of the study include the achievement of "theoretical saturation" or the point when no new data emerged, and the verification of data by a group of mothers not involved in the initial data collection. The theory developed in this study has been based on the experience of first time mothers during the early mothering period. As mothers are challenged by the child's development over the first year of life, the demands of motherhood may change and the process of becoming a mother may evolve. The authors do not specify whether any of the mothers in the study were single; experiences of single mothers may differ from mothers with partners.

The 6 categories of becoming a mother identified in this study are consistent with the findings of other researchers. Previous work highlights the lack of preparation, ${ }^{12}$ loss and isolation, ${ }^{1}$ the importance of social support, ${ }^{3}$ and the interference of fatigue with successful adaptation to the maternal role. ${ }^{14}$

The concepts identified in this study allow predictions of the way individual women are likely to react in early motherhood and highlight the types of interventions that should be designed and evaluated to help women negotiate this experience. An important example of this is the focus on social and relationship issues for new mothers. The emphasis on labour and delivery needs to be balanced with a focus on the magnitude of change which many women encounter in becoming new mothers.

Jane Harvey-Vallender, RGN, RSCN, RHV, MA

Southern Birmingham Community Health National Health Services Trust Birmingham, UK

1 Crouch M, Manderson L. New motherhood; culture and personal transitions. Camberwell: culture and personal transil

2 Tulman L, Fawcett J. Recovery from childbirth: looking back 6 months after delivery. Health Care for Women International. 1991;12:341-50.

3 Oakley A. Social support and motherhood: the natural history of a research project. Oxford: Blackwell, 1992.

4 Tulman L, Fawcett J. Return of functional ability after chidbirth. Nursing Research. 1988;37:
$7-81$. 